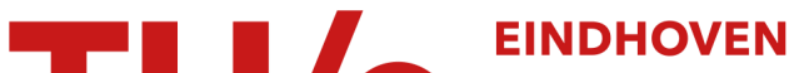 UNIVERSITY OF TECHNOLOGY
}

\section{Dynamic disturbance decoupling for nonlinear systems}

Citation for published version (APA):

Huijberts, H. J. C., Nijmeijer, H., \& vd Wegen, L. L. M. (1992). Dynamic disturbance decoupling for nonlinear systems. SIAM Journal on Control and Optimization, 30(2), 336-349. https://doi.org/10.1137/0330021

DOI:

$10.1137 / 0330021$

Document status and date:

Published: 01/01/1992

\section{Document Version:}

Publisher's PDF, also known as Version of Record (includes final page, issue and volume numbers)

\section{Please check the document version of this publication:}

- A submitted manuscript is the version of the article upon submission and before peer-review. There can be important differences between the submitted version and the official published version of record. People interested in the research are advised to contact the author for the final version of the publication, or visit the $\mathrm{DOI}$ to the publisher's website.

- The final author version and the galley proof are versions of the publication after peer review.

- The final published version features the final layout of the paper including the volume, issue and page numbers.

Link to publication

\section{General rights}

Copyright and moral rights for the publications made accessible in the public portal are retained by the authors and/or other copyright owners and it is a condition of accessing publications that users recognise and abide by the legal requirements associated with these rights.

- Users may download and print one copy of any publication from the public portal for the purpose of private study or research.

- You may not further distribute the material or use it for any profit-making activity or commercial gain

- You may freely distribute the URL identifying the publication in the public portal.

If the publication is distributed under the terms of Article $25 f a$ of the Dutch Copyright Act, indicated by the "Taverne" license above, please follow below link for the End User Agreement:

www.tue.nl/taverne

Take down policy

If you believe that this document breaches copyright please contact us at:

openaccess@tue.nl

providing details and we will investigate your claim. 


\title{
DYNAMIC DISTURBANCE DECOUPLING FOR NONLINEAR SYSTEMS*
}

\author{
H. J. C. HUIJBERTS $\dagger$, H. NIJMEIJER $\dagger$, AND L. L. M. VAN DER WEGEN $\ddagger$
}

\begin{abstract}
In analogy with the dynamic input-output decoupling problem the dynamic disturbance decoupling problem for nonlinear systems is introduced. A local solution of this problem is obtained in the case that the system under consideration is invertible. The solution is given in algebraic as well as in geometric terms. The theory is illustrated by means of two examples: a mathematical one and an example of a voltage frequency controlled induction motor.
\end{abstract}

Key words. nonlinear control systems, dynamic disturbance decoupling, invertibility, dynamic precompensation

AMS(MOS) subject classifications. 93C10, 93B50, 93C35

1. Introduction. Consider a nonlinear multi-input-multi-output control system $\Sigma$ of the form

$$
\Sigma\left\{\begin{array}{l}
\dot{x}=f(x)+g(x) u+p(x) q \\
y=h(x),
\end{array}\right.
$$

where $x \in \mathscr{X}$, an open subset of $\mathbb{R}^{n}$, the inputs $u \in \mathbb{R}^{m}$, the outputs $y \in \mathbb{R}^{p}$, the disturbances $q \in \mathbb{R}^{r}, f$ and $h$ are vector-valued analytic functions, and $g$ and $p$ are matrix-valued analytic functions, all of appropriate dimensions. In the disturbance decoupling problem (DDP) for (1), we search for a regular static state feedback

$$
u=\alpha(x)+\beta(x) v,
$$

with $v$ a new $m$-dimensional control and $\beta(x)$ a nonsingular $m \times m$ matrix for all $x$, so that in the feedback modified dynamics

$$
\dot{x}=f(x)+g(x) \alpha(x)+g(x) \beta(x) v+p(x) q,
$$

the disturbances $q$ do not affect the outputs $y$. A local solution of the DDP using differential geometric tools was initiated in [13] and [9] and has led to a more or less complete understanding of this problem; see, e.g., [12], [18]. The nonlinear DDP forms a direct generalization of the linear DDP, and the theory about the nonlinear DDP typically extends the well-known linear geometric theory (cf. [23]) to a nonlinear context.

The purpose of this paper is to study a dynamic version of the DDP for the nonlinear system (1). That is, instead of a static feedback law (2) we allow for a regular dynamic state feedback

$$
\begin{aligned}
& \dot{z}=\alpha(x, z)+\beta(x, z) v, \\
& u=\gamma(x, z)+\delta(x, z) v,
\end{aligned}
$$

with $z$ the $\mu$-dimensional compensator state and $v$ an $m$-dimensional new control, and the regularity of (4) means that the system (4) with inputs $v$ and outputs $u$ is invertible for all $z$ and constant $x$. Note that a somewhat different definition of regular

\footnotetext{
* Received by the editors January 10, 1990; accepted for publication (in revised form) January 2, 1991.

$\dagger$ Department of Applied Mathematics, University of Twente, Post Office Box 217, 7500 AE Enschede, the Netherlands.

\$ School of Management Studies, University of Twente, Post Office Box 217, 7500 AE Enschede, the Netherlands.
} 
dynamic state feedback was given in [15]. In the dynamic disturbance decoupling problem (DDDP), we require that in the modified dynamics

$$
\begin{aligned}
& \dot{x}=f(x)+g(x) \gamma(x, z)+g(x) \delta(x, z) v+p(x) q, \\
& \dot{z}=\alpha(x, z)+\beta(x, z) v
\end{aligned}
$$

the disturbances $q$ do not influence the outputs $y$. Clearly, the static DDP forms a special case of the DDDP by assuming that $\mu=0$. As noted before, the theory for solving the nonlinear DDP is very much based on a proper extension of the solution of the linear DDP. We are therefore led to think that similarly a solution of the nonlinear DDDP naturally extends the DDDP for linear systems. However, a very simple argument shows that for linear systems the DDDP is solvable if and only if the static DDP is solvable (see, e.g., [1], [2]). Although an analogous result is also true for single-output nonlinear systems, i.e., when $p=1$, this conclusion no longer holds true for multi-output nonlinear systems. In other words, when $p>1$ it may happen that the nonlinear DDDP is (locally) solvable, whereas the nonlinear DDP is not.

Our goal is to establish necessary and sufficient conditions for the solvability of the DDDP, thereby discussing various different algebraic and geometric aspects of this problem for the case that the system (1) with $q \equiv 0$ is square and invertible.

The organization of the paper is as follows. In $\S 2$ we introduce the dynamic disturbance decoupling problem with disturbance measurements (DDDPdm) and the dynamic disturbance decoupling problem (DDDP), and we show that both problems are locally solvable if and only if they are solvable by means of a compensator that is obtained from Singh's algorithm. In $\S \S 3$ and 4 we translate the conditions for solvability of the DDDPdm obtained in $\S 2$ into intrinsic and algorithm-independent conditions, using differential algebraic and geometric tools, respectively. In $\S 5$ the theory of the foregoing sections will be applied to an example of a voltage frequency controlled induction motor as was described in [3]. Section 6 contains the conclusions of the paper.

2. The dynamic disturbance decoupling problem (DDDP). In this section we formulate and solve two kinds of DDDPs. These problems are dynamic extensions of the well-known static state feedback DDP, respectively, the static state feedback DDP with disturbance measurements.

Definition 2.1. Consider the analytic system $\Sigma$ and let a point $x_{0} \in \mathscr{X}$ be given.

1. The DDDP is said to be locally solvable around $x_{0}$ if there exist an analytic dynamic state feedback for $\Sigma$ of the form (4), to be denoted as $R$, with $z \in \mathbb{R}^{\mu}$, a neighborhood $U \subset \mathscr{X}$ of $x_{0}$, and an open subset $\mathscr{Z} \subset \mathbb{R}^{\mu}$ such that $R$, with inputs $v$ and outputs $u$, is invertible for all constants $x \in U$ and $z \in \mathscr{Z}$ and the outputs of the composite system $\Sigma \circ R$ restricted to $U \times \mathscr{Z}$ are independent of the disturbances.

2. The dynamic disturbance decoupling problem with disturbance measurements (DDDPdm) is said to be locally solvable around $x_{0}$ if there exist a dynamic state feedback for $\Sigma$ of the form

$$
Q\left\{\begin{array}{l}
\dot{z}=\alpha(x, q, z)+\beta(x, q, z) v \\
u=\gamma(x, q, z)+\delta(x, q, z) v
\end{array}\right.
$$

with $z \in \mathbb{R}^{\mu}$, a neighborhood $U \subset \mathscr{X}$ of $x_{0}$, and an open subset $\mathscr{Z} \subset \mathbb{R}^{\mu}$, such that (6) with inputs $v$ and outputs $u$ is invertible for all constant $x \in U$ and all $q \in \mathbb{R}^{r}$ and $z \in \mathscr{Z}$, and the outputs of the composite system $\Sigma \circ Q$ restricted to $U \times \mathscr{Z}$ are independent of the disturbances. 
If we furthermore require the compensators $Q$ and $R$ to be static state feedback compensators (i.e., $\mu=0$ ), the problem will be referred to by DDPdm and DDP, respectively.

Recall that the DDP is locally solvable if and only if $\mathscr{P} \subset \Delta^{*}$ and that the DDPdm is locally solvable if and only if $\mathscr{P} \subset \Delta^{*}+\mathscr{G}$, where $\mathscr{G}=\operatorname{span}\left\{g_{1}, \cdots, g_{m}\right\}, \mathscr{P}=$ span $\left\{p_{1}, \cdots, p_{r}\right\}$, and $\Delta^{*}$ is the maximal locally controlled invariant distribution contained in ker $\mathrm{d} h$ (cf. [13]). It is well known (see, e.g., [1], [2]) that for linear systems the DDDP is solvable if and only if the DDP is solvable. That this is not the case for nonlinear systems can be seen from the following example.

Example 2.2. Consider the nonlinear system

$$
\begin{array}{ll}
\dot{x}_{1}=x_{2} u_{1}, & y_{1}=x_{1}, \\
\dot{x}_{2}=x_{5}, & y_{2}=x_{3}, \\
\dot{x}_{3}=x_{2}+x_{4}+x_{4} u_{1}, & \\
\dot{x}_{4}=u_{2}, & \\
\dot{x}_{5}=x_{1} u_{1}+q . &
\end{array}
$$

For this system we have $\Delta^{*}=\{0\}$. Hence the DDP is not locally solvable. However, if we apply the compensator

$$
\begin{gathered}
\dot{z}=v_{1}, \\
u_{1}=z, \\
u_{2}=v_{2},
\end{gathered}
$$

where $v_{1}, v_{2}$ are the new inputs, we find for the compensated system (7), (8) that

$$
\Delta_{e}^{*}=\operatorname{span}\left\{\frac{\partial}{\partial x_{5}}, x_{2}(1+z) \frac{\partial}{\partial x_{2}}+\left(x_{2}-z x_{4}\right) \frac{\partial}{\partial x_{4}}-z(1+z) \frac{\partial}{\partial z}\right\},
$$

where $\Delta_{e}^{*}$ is the maximal locally controlled invariant distribution contained in ker $\mathrm{d} h$ for $(7),(8)$. Hence it is clear that the DDP for (7), (8) is solvable and thus the DDDP is solvable for (7).

In the following, we make the standing assumption, below.

(A1). The system $\Sigma$ is square, i.e., $p=m$.

Instrumental in the solution of the DDDPdm is what we like to call a Singh compensator, which can be obtained via the so-called Singh algorithm. Singh's algorithm has been introduced in [19] for calculation of a left inverse of a nonlinear system. It is a generalization of the algorithm from [8], which was only applicable under some restrictive assumptions. Let $\Sigma_{0}$ denote the system $\Sigma$ with $q \equiv 0$. We briefly repeat Singh's algorithm for the system $\Sigma_{0}$, following [5].

Algorithm 2.3. Consider the analytic nonlinear system $\Sigma_{0}$, satisfying (A1). Let a point $x_{0} \in \mathscr{X}$ be given.

Step 1.

Calculate

$$
\dot{y}=\frac{\partial h}{\partial x}[f(x)+g(x) u]=: a_{1}(x)+b_{1}(x) u
$$


and assume that $b_{1}(x)$ has full rank $\rho_{1}$ on a neighborhood of $x_{0}$. Define $s_{1}:=\rho_{1}$. Permute, if necessary, the components of the output, so that the first $\rho_{1}$ rows of $b_{1}(x)$ are linearly independent. Decompose y according to

$$
\dot{y}=\left(\begin{array}{c}
\dot{\hat{y}}_{1} \\
\dot{\hat{y}_{1}}
\end{array}\right) \text {, }
$$

where $\dot{y}_{1}$ consists of the first $\rho_{1}$ rows of $\dot{y}$. Since the last rows of $b_{1}(x)$ are linearly dependent on the first $\rho_{1}$ rows, we can write

$$
\tilde{y}_{1}=\tilde{a}_{1}(x)+\tilde{b}_{1}(x) u, \quad \dot{\hat{y}}_{1}=\dot{\hat{y}}_{1}\left(x, \dot{\tilde{y}}_{1}\right),
$$

where the last equation is affine in $\tilde{y}_{1}$. Finally, set $\tilde{B}_{1}(x):=\tilde{b}_{1}(x)$.

Step $k+1$.

Suppose that in Steps 1 through $k, \tilde{y}_{1}, \cdots, \tilde{y}_{k}^{(k)}, \hat{y}_{k}^{(k)}$ have been defined so that

$$
\begin{aligned}
\tilde{y}_{1}= & \tilde{a}_{1}(x)+\tilde{b}_{1}(x) u, \\
& \vdots \\
\tilde{y}_{k}^{(k)}= & \tilde{a}_{k}\left(x,\left\{\tilde{y}_{i}^{(j)} \mid 1 \leqq i \leqq k-1, i \leqq j \leqq k\right\}\right) \\
& +\tilde{b}_{k}\left(x,\left\{\tilde{y}_{i}^{(j)} \mid 1 \leqq i \leqq k-1, i \leqq j \leqq k-1\right\}\right) u, \\
\hat{y}_{k}^{(k)}= & \hat{y}_{k}^{(k)}\left(x,\left\{\tilde{y}_{i}^{(j)} \mid 1 \leqq i \leqq k, i \leqq j \leqq k\right\}\right) .
\end{aligned}
$$

Suppose also that there exist $\tilde{y}_{i 0}^{(j)}(1 \leqq i \leqq k-1, i \leqq j \leqq k-1)$ such that the matrix $\tilde{B}_{k}:=$ $\left[\tilde{b}_{1}^{T}, \cdots, \tilde{b}_{k}^{T}\right]^{T}$ has full rank $\rho_{k}$ on a neighborhood of $\left(x_{0},\left\{\tilde{y}_{i 0}^{(j)} \mid 1 \leqq i \leqq k-1, i \leqq j \leqq k-\right.\right.$ 1\}). Then calculate

$$
\hat{y}_{k}^{(k+1)}=\frac{\partial}{\partial x} \hat{y}_{k}^{(k)}[f(x)+g(x) u]+\sum_{i=1}^{k} \sum_{j=i}^{k} \frac{\partial \hat{y}_{k}^{(k)}}{\partial \tilde{y}_{i}^{(j)}} \tilde{y}_{i}^{(j+1)}
$$

and write it as

$$
\begin{aligned}
\hat{y}_{k}^{(k+1)}= & a_{k+1}\left(x,\left\{\tilde{y}_{i}^{(j)} \mid 1 \leqq i \leqq k, i \leqq j \leqq k+1\right\}\right) \\
& +b_{k+1}\left(x,\left\{\tilde{y}_{i}^{(j)} \mid 1 \leqq i \leqq k, i \leqq j \leqq k\right\}\right) u .
\end{aligned}
$$

Define $B_{k+1}:=\left[\tilde{B}_{k}^{T}, b_{k+1}^{T}\right]^{T}$, and suppose that there exist $\tilde{y}_{i 0}^{(j)}(1 \leqq i \leqq k, i \leqq j \leqq k)$ such that $B_{k+1}$ has constant rank $\rho_{k+1}$ on a neighborhood of $\left(x_{0},\left\{\tilde{y}_{i 0}^{(j)} \mid 1 \leqq i \leqq k, i \leqq j \leqq k\right\}\right)$. Permute, if necessary, the components of $\hat{y}_{k}^{(k+1)}$ so that on this neighborhood the first $\rho_{k+1}$ rows of $B_{k+1}$ are linearly independent. Decompose $\hat{y}_{k}^{(k+1)}$ as

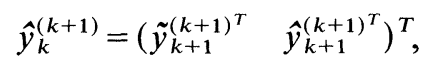

where $\tilde{y}_{k+1}^{(k+1)}$ consists of the first $s_{k+1}:=\left(\rho_{k+1}-\rho_{k}\right)$ rows. Since the last rows of $B_{k+1}$ are linearly dependent on the first $\rho_{k+1}$ rows, we can write

$$
\begin{aligned}
\tilde{y}_{1}= & \tilde{a}_{1}(x)+\tilde{b}_{1}(x) u, \\
& \vdots \\
\tilde{y}_{k+1}^{(k+1)}= & \tilde{a}_{k+1}\left(x,\left\{\tilde{y}_{i}^{(j)} \mid 1 \leqq i \leqq k, i \leqq j \leqq k+1\right\}\right) \\
& +\tilde{b}_{k+1}\left(x,\left\{\tilde{y}_{i}^{(j)} \mid 1 \leqq i \leqq k, i \leqq j \leqq k\right\}\right) u, \\
\hat{y}_{k+1}^{(k+1)}= & \hat{y}_{k+1}^{(k+1)}\left(x,\left\{\tilde{y}_{i}^{(j)} \mid 1 \leqq i \leqq k+1, i \leqq j \leqq k+1\right\}\right) .
\end{aligned}
$$

Finally, set $\tilde{B}_{k+1}:=\left[\tilde{B}_{k}^{T}, \tilde{b}_{k+1}^{T}\right]^{T}$.

It should be noted that the integers $\rho_{1}, \cdots, \rho_{k}, \cdots$, defined above, do not depend on the particular permutation of the rows of $\hat{y}_{k}^{(k+1)}$ we employ, cf. [5]. So, using the 
algorithm we obtain a uniquely defined sequence of integers $0 \leqq \rho_{1} \leqq \cdots \leqq \rho_{k} \leqq \cdots \leqq m$. The integer $\rho^{*}:=\rho_{n}$ is called the rank of the system (1), cf. [18], [5]. We associate a notion of regularity with Singh's algorithm in the following way. (See [4] for a slightly different notion of regularity.)

DEFINITION 2.4. Let a point $x_{0} \in \chi$ be given. We call $x_{0}$ a strongly regular point for $\Sigma$ if, for each application of Singh's algorithm to $\Sigma_{0}$, the constant rank assumptions of the algorithm are satisfied.

Besides (A1), we also introduce the following assumption.

(A2). The system $\Sigma_{0}$ is invertible, i.e., $\rho^{*}=m$.

Consider a system $\Sigma$ satisfying (A1), (A2). Then we define a Singh compensator for $\Sigma$ as follows (see also [20]). Let $x_{0}$ be a strongly regular point for $\Sigma$ and apply Singh's algorithm for $\Sigma_{0}$. This yields at the $n$th step

$$
\begin{aligned}
\dot{y}_{n}= & \tilde{A}_{n}\left(x,\left\{\tilde{y}_{i}^{(j)} \mid 1 \leqq i \leqq n-1, i \leqq j \leqq n\right\}\right) \\
& +\tilde{B}_{n}\left(x,\left\{\tilde{y}_{i}^{(j)} \mid 1 \leqq i \leqq n-1, i \leqq j \leqq n-1\right\}\right) u,
\end{aligned}
$$

where $\tilde{Y}_{n}=\left(\tilde{y}_{1}^{T}, \cdots, \tilde{y}_{n}^{(n-1)^{T}}\right)$ and where $\tilde{B}_{n}$ is invertible on a neighborhood of $\left(x_{0},\left\{\tilde{y}_{i 0}^{(j)} \mid 1 \leqq i \leqq n-1, i \leqq j \leqq n-1\right\}\right)$ for some $\tilde{y}_{i 0}^{(j)}(1 \leqq i \leqq n, i \leqq j \leqq n)$. Then (16) yields on this neighborhood

$$
u=\tilde{B}_{n}^{-1}\left[\hat{Y}_{n}-\tilde{A}_{n}\right]
$$

For $i=1, \cdots, m$, let $\gamma_{i}$ be the lowest time-derivative and $\delta_{i}$ be the highest time-derivative of $y_{i}$ appearing in (17). Then we rewrite (17) as

$$
\begin{aligned}
u= & \phi_{1}\left(x,\left\{y_{i}^{(j)} \mid 1 \leqq i \leqq m, \gamma_{i} \leqq j \leqq \delta_{i}-1\right\}\right) \\
& +\sum_{i=1}^{m} \phi_{2 i}\left(x,\left\{y_{i}^{(j)} \mid 1 \leqq i \leqq m, \gamma_{i} \leqq j \leqq \delta_{i}-1\right\}\right) y_{i}^{\left(\delta_{i}\right)}
\end{aligned}
$$

for certain locally analytic vector-valued functions $\phi_{1}, \phi_{2 i}(i=1, \cdots, m)$.

Let $z_{i}(i=1, \cdots, m)$ be a vector of dimension $\delta_{i}-\gamma_{i}$ and consider the system

$$
\begin{aligned}
\dot{z}_{i} & =A_{i} z_{i}+B_{i} v_{i}(i=1, \cdots, m), \\
u & =\phi_{1}\left(x, z_{1}, \cdots, z_{m}\right)+\sum_{i=1}^{m} \phi_{2 i}\left(x, z_{1}, \cdots, z_{m}\right) v_{i},
\end{aligned}
$$

with inputs $v_{1}, \cdots, v_{m}$, outputs $u,\left(A_{i}, B_{i}\right)$ in Brunovsky canonical form, and $z_{i 0}=$ $\left(y_{i 0}^{\left(\gamma_{i}\right)}, \cdots, y_{i 0}^{\left(\delta_{i}-1\right)}\right)^{T}(i=1, \cdots, m)$. Then (19) is called a Singh compensator for $\Sigma$ around $x_{0}$. Note that the Singh compensator (19) is an inverse of the system $\Sigma_{0}$ around $x_{0}$.

Remark 2.5. The Singh compensator, constructed above, has dimension $\sigma=$ $\sum_{i=1}^{m}\left(\delta_{i}-\gamma_{i}\right)$. It can be shown (see [11]) that every Singh compensator has dimension $\sigma$. Moreover, the numbers $\delta_{i}(i=1, \cdots, m)$ are equal to the essential orders (cf. [7]) of $\Sigma$ (see also [11]).

We obtain a Singh compensator with disturbance feedthrough for $\Sigma$ around $x_{0}$ by extending (19) in the following way. Define in the above notation

$$
\phi_{3}\left(x,\left\{y_{i}^{(j)} \mid 1 \leqq i \leqq m, \gamma_{i} \leqq j \leqq \delta_{i}-1\right\}\right):=-\tilde{B}_{n}^{-1} \frac{\partial \tilde{Y}_{n}}{\partial x} p(x)
$$

and consider the following extension of (19):

$$
\begin{aligned}
\dot{z}_{i} & =A_{i} z_{i}+B_{i} v_{i}(i=1, \cdots, m), \\
u & =\phi_{1}\left(x, z_{1}, \cdots, z_{m}\right)+\sum_{i=1}^{m} \phi_{2 i}\left(x, z_{1}, \cdots, z_{m}\right) v_{i}+\phi_{3}\left(x, z_{1}, \cdots, z_{m}\right) q .
\end{aligned}
$$


Then (21) is called a Singh compensator with disturbance feedthrough for $\Sigma$ around $x_{0}$. It can be shown that the Singh compensator as well as the Singh compensator with disturbance feedthrough constitute a regular dynamic state feedback (cf. [10]).

We will now state our main result. In this statement we employ the following notation. If we apply Singh's algorithm to $\Sigma_{0}$, the $\hat{y}_{k}^{(k)}\left(k=0, \cdots, n ; \hat{y}_{0}:=y\right)$ can be viewed as functions on $\mathscr{X}_{e}:=\mathscr{X} \times \mathbb{R}^{n m}$. By the same token, $\operatorname{ker} \mathrm{d} \hat{y}_{k}^{(k)}(k=0, \cdots, n)$ defines a distribution on $\mathscr{X}_{e}$. Define the distributions $\mathscr{G}_{e}, \mathscr{P}_{e}$ on $\mathscr{X}_{e}$ by $\mathscr{G}_{e}:=\mathscr{G} \times\{0\}, \mathscr{P}_{e}:=\mathscr{P} \times\{0\}$.

THEOREM 2.6. Consider the analytic system $\Sigma$. Assume that it satisfies (A1), (A2) and that $x_{0}$ is a strongly regular point for $\Sigma$.

1. (a) The DDDP is locally solvable around $x_{0}$ if and only if it is solvable via a Singh compensator for $\Sigma$ around $x_{0}$.

(b) The above condition is equivalent to the condition that for each application of Singh's algorithm to $\Sigma_{0}$ we have for $k=0, \cdots, n-1$ :

$$
\mathscr{P}_{e} \subset \operatorname{kerd} \hat{y}_{k}^{(k)} \text {. }
$$

2. (a) The DDDPdm is locally solvable around $x_{0}$ if and only if it is solvable via a Singh compensator with disturbance feedthrough for $\Sigma$ around $x_{0}$.

(b) the above condition is equivalent to the condition that for each application of Singh's algorithm to $\Sigma_{0}$ we have, for $k=0, \cdots, n-1$,

$$
\mathscr{P}_{e} \subset \operatorname{ker} \mathrm{d} \hat{y}_{k}^{(k)}+\mathscr{G}_{e} \text {. }
$$

Proof. We will prove only part 2 . The proof of part 1 is analogous.

Sufficiency. Consider $\Sigma$ and assume that it satisfies (A1), (A2), and let $x_{0}$ be a strongly regular point for $\Sigma$. Assume that for each application of Singh's algorithm to $\Sigma_{0}(23)$ holds for $k=0, \cdots, n-1$. Apply Singh's algorithm to $\Sigma_{0}$ around $x_{0}$, yielding a reordering $\tilde{y}_{1}, \cdots, \tilde{y}_{n}$ of the outputs. Note that, without loss of generality, we may assume that for $k=1, \cdots, n: \hat{y}_{k}=\left(\tilde{y}_{k+1}^{T}, \cdots, \tilde{y}_{n}^{T}\right)^{T}$. The first step of Singh's algorithm applied to $\Sigma_{0}$ around $x_{0}$ yields

$$
\begin{aligned}
& \hat{y}_{1}=\tilde{a}_{1}(x)+\tilde{b}_{1}(x) u, \\
& \dot{\hat{y}}_{1}=\hat{a}_{1}(x)+\hat{b}_{1}(x) u,
\end{aligned}
$$

where $\tilde{b}_{1}(x)$ has full row rank $\rho_{1}$ on a neighborhood $U$ of $x_{0}$ in $\mathscr{X}$. Let $\tilde{b}_{1}^{+}(x)$ be a right inverse of $\tilde{b}_{1}(x)$ on $U$. Then on $U, \dot{\hat{y}}_{1}$ takes the form

$$
\dot{\hat{y}}_{1}=\hat{a}_{1}(x)+\hat{b}_{1}(x) \hat{b}_{1}^{+}(x)\left(\dot{y}_{1}-\tilde{a}_{1}(x)\right) \text {. }
$$

Note that, since on $U$ each of the rows of $\hat{b}_{1}(x)$ is linearly dependent on the rows of $\tilde{b}_{1}(x)$, the form of $\dot{y}_{1}$ is independent of the choice of $\tilde{b}_{1}^{+}(x)$. For $\Sigma$ we have

$$
\begin{aligned}
& \dot{y}_{1}=\frac{\partial \tilde{y}_{1}}{\partial x}[f(x)+g(x) u+p(x) q]=: \tilde{a}_{1}(x)+\tilde{b}_{1}(x) u+\tilde{c}_{1}(x) q, \\
& \dot{\hat{y}}_{1}=\frac{\partial \hat{y}_{1}}{\partial x}[f(x)+g(x) u+p(x) q]=: \hat{a}_{1}(x)+\hat{b}_{1}(x) u+\hat{c}_{1}(x) q,
\end{aligned}
$$

with $\tilde{a}_{1}(x), \tilde{b}_{1}(x), \hat{a}_{1}(x), \hat{b}_{1}(x)$ as in (24). It can easily be checked that the fact that (23) holds for $k=0$ is equivalent to the existence of a $\sigma_{1}(x)$ such that

$$
\tilde{b}_{1}(x) \sigma_{1}(x)=\tilde{c}_{1}(x), \hat{b}_{1}(x) \sigma_{1}(x)=\hat{c}_{1}(x) .
$$

Then (26) and (27) yield

$$
\dot{\hat{y}}_{1}=\hat{a}_{1}(x)+\hat{b}_{1}(x) \tilde{b}_{1}^{+}(x)\left(\dot{y}_{1}-\tilde{a}_{1}(x)\right) .
$$


Hence for $\Sigma, \dot{\hat{y}}_{1}$ is given by the same expression as for $\Sigma_{0}$. Applying the above arguments repeatedly, we can show that for $\Sigma \hat{y}_{k}^{(k)}(k=1, \cdots, n)$ has the same form as for $\Sigma_{0}$, and that for $\Sigma, \hat{\tilde{Y}}_{n}$ takes the form (see also (16))

$$
\tilde{Y}_{n}=\tilde{A}_{n}+\tilde{B}_{n} u+\frac{\partial \hat{Y}_{n}}{\partial x} p(x) q .
$$

This implies that if we apply the Singh compensator with disturbance feedthrough to $\Sigma$, the outputs of the resulting system satisfy

$$
\begin{aligned}
\frac{\partial y_{i}^{(j)}}{\partial q} & =0 \quad\left(1 \leqq i \leqq m, 0 \leqq j \leqq \delta_{i}-1\right), \\
y_{i}^{\left(\delta_{i}\right)} & =v_{i} .
\end{aligned}
$$

Hence the Singh compensator with disturbance feedthrough locally solves the DDDPdm for $\Sigma$ around $x_{0}$.

Necessity. Assume that the DDDPdm is locally solvable around $x_{0}$ by means of a compensator $Q$ of the form (6). Apply Singh's algorithm to $\Sigma_{0}$ around $x_{0}$, yielding a reordering $\tilde{y}_{1}, \cdots, \tilde{y}_{n}$ of the outputs. Then with the notation of the necessity part of this proof, we have in particular for $\Sigma$

$$
\begin{aligned}
& \dot{y}_{1}=\tilde{a}_{1}(x)+\tilde{b}_{1}(x) u+\tilde{c}_{1}(x) q, \\
& \dot{\hat{y}}_{1}=\hat{a}_{1}(x)+\hat{b}_{1}(x) \tilde{b}_{1}^{+}(x)\left(\dot{y}_{1}-\tilde{a}_{1}(x)\right)+\left(\hat{c}_{1}(x)-\hat{b}_{1}(x) \tilde{b}_{1}^{+}(x) \tilde{c}_{1}(x)\right) q .
\end{aligned}
$$

Assume that (23) does not hold for $k=0$. This implies that $d_{1}(x):=$ $\hat{c}_{1}(x)-\hat{b}_{1}(x) \tilde{b}_{1}^{+}(x) \tilde{c}_{1}(x) \not \equiv 0$. Since $Q$ solves the DDDPdm for $\Sigma$, the $q$-dependence in (31) should disappear if we plug the output of $Q$ in (31). From the form of (31), it is clear that this is only possible if $Q$ imposes the constraint $d_{1}(x)=0$ on the system $\Sigma \circ Q$. However, this would imply that the DDDPdm is at most solvable on a neighborhood of $x_{0}$ in $\left\{x \mid d_{1}(x)=0\right\}$ and not on a neighborhood of $x_{0}$ in $\mathscr{X}$. Hence we must necessarily have that (23) holds for $k=0$. Next assume that (23) does not hold for $k=1$. Then by the same arguments as above we will have for $\Sigma$ that $\hat{y}_{2}^{(2)}$ ) explicitly depends on $q$ via a function $d_{2}\left(x, \dot{y}_{1}\right)$ and that this $q$-dependence can only disappear if $Q$ imposes the constraint $d_{2}\left(x, \dot{\tilde{y}}_{1}\right)=0$ on the system. However, by Lemma 1 of [14], this would imply that the rank of $\Sigma \circ Q$ is smaller that the rank of $\Sigma$, which would contradict the invertibility of $Q$. Therefore (23) must hold for $k=1$. Applying this argument repeatedly, we show that (23) holds for $k=0, \cdots, n-1$, which establishes our claim. By the sufficiency part of this proof, this also immediately implies that we can solve the DDDPdm around $x_{0}$ via a Singh compensator with disturbance feedthrough.

Example 2.2 (continued). The Singh compensator

$$
\begin{aligned}
\dot{z} & =v_{1}, \\
u_{1} & =\frac{z}{x_{2}}, \\
u_{2} & =\frac{x_{5}\left(x_{4} z-x_{2}\right)}{x_{2}+z}-\frac{x_{4}}{x_{2}+z} v_{1}+\frac{x_{2}}{x_{2}+z} v_{2},
\end{aligned}
$$

solves the DDDP for system (7) of the earlier part of Example 2.2.

Remark 2.7. It is easily seen that if the input-output decoupling problem for $\Sigma$ is locally solvable by means of a static state feedback, then the DDDPdm is locally 
solvable if and only if the DDDPdm is. This implies in particular that if the output of $\Sigma$ is one-dimensional, then the DDDPdm is locally solvable if and only if the DDPdm is.

3. Algebraic conditions. In this section we translate the conditions for solvability of the DDDPdm and the DDDP obtained in $\S 2$ into intrinsic and algorithm-independent conditions. It will turn out that the conditions can be stated in terms of a certain structure at infinity.

For nonlinear systems there are several different definitions of the structure at infinity; see, e.g., [17], [14]. We call the structure at infinity that was defined in [17] the geometric structure at infinity. This structure at infinity has proved its importance in, e.g., the solution of the static state feedback input-output decoupling problem (cf. [17]) and in obtaining (sufficient) conditions for solvability of the nonlinear model matching problem (cf. [6]). Here we will use the algebraic definition of [14], which in general yields a different structure at infinity than the geometric one. This structure at infinity has already proved its importance in the dynamic state feedback input-output decoupling problem and in obtaining sufficient conditions for solvability of the nonlinear model matching problem (cf. [16]) that are weaker than the conditions obtained in [6]. To repeat this algebraic definition, we consider Singh's algorithm as described in $\S 2$ and define the following integers:

$$
\begin{aligned}
\pi_{1} & :=\rho^{*}, \\
\pi_{k} & :=\rho^{*}-\rho_{k-1}, \quad k \geqq 2, \\
\nu_{i} & :=\text { the number of } \pi_{k} \text { 's that are greater than or equal to } i, \quad i \geqq 1 .
\end{aligned}
$$

Definition 3.1 (see [14]). The algebraic structure at infinity of the system $\Sigma_{0}$ consists of the set $\left\{\nu_{i}\right\}$ of orders of the zeros at infinity, together with the set $\left\{\pi_{k}\right\}$ of numbers of zeros at infinity whose order is greater than or equal to $k$.

Essentially, the sets $\left\{\nu_{i}\right\},\left\{\pi_{k}\right\}$, and $\left\{\rho_{k}\right\}$ contain the same information about the algebraic structure at infinity. In the following, we restrict ourselves to considering the set $\left\{\rho_{k}\right\}$.

Let $\Sigma_{q}$ denote the system $\Sigma$ where the disturbances are considered to be an extra set of inputs. For $\Sigma_{0}$ and $\Sigma_{q}$, the sets defining their algebraic structure at infinity are denoted by $\left\{\rho_{0 k}\right\},\left\{\rho_{q k}\right\}$, respectively.

THEOREM 3.2. Consider the analytic system $\Sigma$ and assume that it satisfies (A1), (A2). Let $x_{0}$ be a strongly regular point for $\Sigma$. Then the DDDPdm is locally solvable around $x_{0}$ if and only if $\Sigma_{q}$ and $\Sigma_{0}$ have the same algebraic structure at infinity.

Proof. Consider the first step of Singh's algorithm applied to $\Sigma_{0}$ and $\Sigma_{q}$ around $x_{0}$ and employ the notation of the sufficiency part of the proof of Theorem 2.6. As was shown in this proof, condition (23) is satisfied for $k=0$ if and only if there exists a $\sigma_{1}(x)$ such that (27) holds. Hence the fact that (23) holds for $k=0$ is equivalent to

$$
\rho_{q 1}=\operatorname{rank}\left(\begin{array}{cc}
\tilde{b}_{1} & \tilde{c}_{1} \\
\hat{b}_{1} & \hat{c}_{1}
\end{array}\right)=\operatorname{rank}\left(\begin{array}{cc}
\tilde{b}_{1} & \tilde{b}_{1} \sigma_{1} \\
\hat{b}_{1} & \hat{b}_{1} \sigma_{1}
\end{array}\right)=\operatorname{rank}\left(\begin{array}{l}
\tilde{b}_{1} \\
\hat{b}_{1}
\end{array}\right)=\rho_{01} .
$$

Applying the above arguments repeatedly, we show that (23) holds for $k=0, \cdots, n-1$ if and only if $\rho_{q k}=\rho_{0 k}$ for $k=1, \cdots, n$, which establishes our claim.

Remark 3.3. Recall that for linear systems the geometric and algebraic structure at infinity are the same (cf. [14]). Moreover, recall from, e.g., [22] that for linear systems the DDPdm is solvable if and only if $\Sigma_{0}$ and $\Sigma_{q}$ have the same structure at infinity. 
We will now use the result of Theorem 3.2 to give conditions for the local solvability of the DDDP for $\Sigma$, using an idea from [22]. To do this we introduce an auxiliary system $\Sigma_{a}$, which is defined as

$$
\Sigma_{a}\left\{\begin{array}{l}
\dot{x}=f(x)+g(x) w+p(x) q \\
\dot{w}=v \\
y=h(x)
\end{array}\right.
$$

where $v$ denotes the inputs of $\Sigma_{a}$. Let $\Sigma_{a 0}$ denote the system $\Sigma_{a}$ with $q \equiv 0$ and let $\Sigma_{a q}$ denote the system $\Sigma_{a}$ where the disturbances are considered to be an extra set of inputs.

THEOREM 3.4. Consider the analytic system $\Sigma$ and assume that it satisfies (A1), (A2). Let $x_{0}$ be a strongly regular point for $\Sigma$. Then the DDDP is locally solvable around $x_{0}$ if and only if $\Sigma_{a q}$ and $\Sigma_{a 0}$ have the same algebraic structure at infinity.

Proof. Necessity. Assume that the DDDP is locally solvable around $x_{0}$ by means of a compensator

$$
R\left\{\begin{array}{l}
\dot{z}=\alpha(x, z)+\beta(x, z) \tilde{u} \\
u=\gamma(x, z)+\delta(x, z) \tilde{u}
\end{array}\right.
$$

Consider the following compensator for $\Sigma_{a}$ :

$$
R_{a}\left\{\begin{array}{l}
\dot{z}_{1}=\alpha\left(x, z_{1}\right)+\beta\left(x, z_{1}\right) z_{2} \\
\dot{z}_{2}=\hat{u} \\
v=\sigma\left(x, w, z_{1}, z_{2}, q\right),
\end{array}\right.
$$

with

$$
\begin{aligned}
\sigma\left(x, w, z_{1}, z_{2}, q\right)= & {\left[\frac{\partial \gamma}{\partial x}\left(x, z_{1}\right)+\frac{\partial \delta}{\partial x}\left(x, z_{1}\right) z_{2}\right][f(x)+g(x) w+p(x) q] } \\
& +\left[\frac{\partial \gamma}{\partial z_{1}}\left(x, z_{1}\right)+\frac{\partial \delta}{\partial z_{1}}\left(x, z_{1}\right) z_{2}\right]\left[\alpha\left(x, z_{1}\right)+\beta\left(x, z_{1}\right) z_{2}\right]+\delta\left(x, z_{1}\right) \hat{u}
\end{aligned}
$$

and $\hat{u}$ denoting the new inputs. Then we find that

$$
w=\int v d t=\gamma\left(x, z_{1}\right)+\delta\left(x, z_{1}\right) z_{2}
$$

and thus $R_{a}$ locally solves the DDDPdm for $\Sigma_{a}$, since $R$ solves the DDDP for $\Sigma$. Furthermore, we have as an (almost) immediate consequence of the fact that $R$ is invertible, that $R_{a}$ is also invertible. Thus, the DDDPdm is locally solvable for $\Sigma_{a}$ and hence by Theorem 3.2, $\Sigma_{a q}$ and $\Sigma_{a 0}$ have the same algebraic structure at infinity.

Sufficiency. Assume that $\Sigma_{a q}$ and $\Sigma_{a 0}$ have the same algebraic structure at infinity; i.e., the DDDPdm is locally solvable for $\Sigma_{a}$, say, by means of a compensator

$$
Q_{a}\left\{\begin{array}{l}
\dot{z}=\alpha(x, w, z, q)+\beta(x, w, z, q) s \\
v=\gamma(x, w, z, q)+\delta(x, w, z, q) s
\end{array}\right.
$$

with $s$ denoting the new inputs. Then it is obvious that the compensator

$$
Q\left\{\begin{array}{l}
\dot{z}_{1}=\gamma\left(x, z_{1}, z_{2}, q\right)+\delta\left(x, z_{1}, z_{2}, q\right) s \\
\dot{z}_{2}=\alpha\left(x, z_{1}, z_{2}, q\right)+\beta\left(x, z_{1}, z_{2}, q\right) s \\
u=z_{1}
\end{array}\right.
$$


locally solves the DDDPdm for $\Sigma$. Now apply Singh's algorithm to $\Sigma_{0}$, yielding a reordering $\tilde{y}_{1}, \cdots, \tilde{y}_{n}$ of the outputs. Employ the notation of the proof of Theorem 2.6. Then for $\Sigma$ we have in particular that

$$
\begin{aligned}
& \dot{y}_{1}=\tilde{a}_{1}(x)+\tilde{b}_{1}(x) u+\tilde{c}_{1}(x) q, \\
& \dot{\hat{y}}_{1}=\hat{a}_{1}(x)+\hat{b}_{1}(x) u+\hat{c}_{1}(x) q .
\end{aligned}
$$

Since $Q$ locally solves the DDDPdm for $\Sigma$, the $q$-dependence in (40) must have vanished if we put $u$ in (40) equal to the output of $Q$. This implies that actually $\tilde{c}_{1} \equiv 0$, $\hat{c}_{1} \equiv 0$, since the output of $Q$ does not depend on $q$. It can be checked that this implies that (22) holds for $k=0$. Applying the above arguments repeatedly, we can show that (22) holds for $k=0, \cdots, n-1$. By Theorem 2.6 this implies that the DDDP is solvable around $x_{0}$.

Remark 3.5. At this point it is useful to compare our (algebraic) results on the DDDP with the results of [16] on the so-called nonlinear model matching problem (MMP). In fact, as was already shown in [6], the nonlinear MMP can be related to some kind of DDPdm. However, in contrast with the situation we consider in this paper, it turns out that the corresponding state feedback solving this DDPdm need not be regular. Clearly, the existence of a regular solution is, of course, a sufficient condition for solvability of the nonlinear MMP. Hence by Theorem 3.2 the coincidence of certain algebraic structures at infinity is a sufficient condition for solvability of the nonlinear MMP. This is exactly the result of [16]. As the equality of the algebraic structures at infinity is only a sufficient condition for the solvability of the MMP, and not a necessary condition (see [16] for a counterexample), it is clear that our solution of the DDDPdm by means of regular dynamic state feedback cannot be cast in the results described in the aforementioned paper.

4. Geometric conditions. In this section we give intrinsic geometric conditions for local solvability of the DDDP and the DDDPdm by translating the results of $\S 3$. We mainly restrict our attention to the DDDP. The reasoning for the DDDPdm follows the same lines.

Consider again system $\Sigma$ and assume that it satisfies (A1), (A2). Let $x_{0}$ be a strongly regular point for $\Sigma$. Furthermore, assume that the DDDP for $\Sigma$ is locally solvable around $x_{0}$. Then according to Theorem 2.6 the DDDP is locally solvable by applying a Singh compensator. This compensator applied to $\Sigma$ yields $y_{i}^{\left(\delta_{i}\right)}=v_{i}, i=1, \cdots, m$. Obviously, the decoupling matrix (see [12], [18]) is equal to the $m \times m$ identity matrix. Hence the decoupling matrix of the composite system is of full rank. Then it is well known that the maximal locally controlled invariant distribution in ker $\mathrm{d} h$ for the composite system, denoted by $\Delta_{e}^{*}$, is given by

$$
\Delta_{e}^{*}=\bigcap_{i=1}^{m} \bigcap_{k=0}^{\delta_{i}-1} \operatorname{ker} \mathrm{d} y_{i}^{(k)} .
$$

Obviously, $\Delta_{e}^{*}$ depends on the choice of the $\tilde{y}_{k}^{(k)}$ 's in Singh's algorithm, so $\Delta_{e}^{*}$ is by no means uniquely defined. However, the solvability of the DDDP does not depend on the way in which Singh's algorithm is performed (see Theorem 2.6). Hence, for any distribution $\Delta$ of the form (41) generated by applying Singh's algorithm to $\Sigma_{0}$, we have $\mathscr{P} \times\{0\} \subset \Delta$. Consequently, the distribution $\mathscr{P} \times\{0\}$ spanned by the extended disturbance vector fields is always contained in $\Delta_{e}^{*}$. Note that by construction $\Delta_{e}^{*}$ is contained in $T \mathscr{X} \times\{0\} \subset T \mathscr{X} \times T \mathscr{Z}$ (with abuse of notation). However, the vector fields that span $\Delta_{e}^{*}$ may very well depend on $z$. Since the disturbance vector fields $p$ only depend on 
$x$, they are contained in the (not necessarily controlled invariant) maximal subdistribution $\tilde{\Delta}_{e}^{*}$ of $\Delta_{e}^{*}$ that contains the vector fields in $\Delta_{e}^{*}$ that only depend on $x$. This distribution $\tilde{\Delta}_{e}^{*}$ can be found by means of the following algorithm.

Algorithm 4.1.

Step 1.

$$
\Delta_{0}:=\Delta_{e}^{*}
$$

Step $k$.

$$
\Delta_{k}:=\left\{\tau \in \Delta_{k-1} \mid\left[\tau, \frac{\partial}{\partial z}\right] \in \Delta_{k-1}+\operatorname{span}\left\{\frac{\partial}{\partial z}\right\}\right\} .
$$

Here $[\tau, \partial / \partial z]$ is shorthand notation for the Lie-brackets $\left[\tau, \partial / \partial z_{i}\right]$ for $i=1, \cdots, \sigma$.

Lemma 4.2. Assume that the distributions $\Delta_{k}$ obtained in Algorithm 4.1 have constant dimension. Then for all $k, \operatorname{dim} \Delta_{k} \leqq \operatorname{dim} \Delta_{k-1}$ and if $\Delta_{k^{*}}=\Delta_{k^{*-1}}$, then $\Delta_{k}=\Delta_{k^{*}}$ for all $k \geqq k^{*}$.

Proof. See [12].

Assume now that Algorithm 4.1 converges to $\Delta_{k^{*}}$. Then $\Delta_{k^{*}}$ fulfills the condition

$$
\left[\Delta_{k^{*}}, \frac{\partial}{\partial z}\right] \subset \Delta_{k^{*}}+\operatorname{span}\left\{\frac{\partial}{\partial z}\right\} \text {. }
$$

As can be seen in [21], it follows from (42) that the first $n$ components of any vector field in $\Delta_{k^{*}}$ do not depend on $z$, and since $\Delta_{k^{*}} \subset \Delta_{e}^{*}$ (so the last $\sigma$ components of vector fields in $\Delta_{k^{*}}$ equal zero) the vector fields in $\Delta_{k^{*}}$ do not depend on $z$ at all. Moreover, by construction, $\Delta_{k^{*}}$ is the largest subdistribution of $\Delta_{e}^{*}$ having this property. Hence $\Delta_{k^{*}}=\tilde{\Delta}_{e}^{*}$.

LEMMA 4.3. $\tilde{\Delta}_{e}^{*}$ obtained in the way described above is independent of the way we apply Singh's algorithm.

Proof. Assume we have applied Singh's algorithm in two different ways, yielding $\Delta_{e 1}^{*}$ and $\Delta_{e 2}^{*}$. Assume furthermore that by applying Algorithm 4.1 to these two distributions we obtain the distributions $\tilde{\Delta}_{e 1}^{*}$ and $\tilde{\Delta}_{e 2}^{*}$ with $\tilde{\Delta}_{e 1}^{*} \neq \tilde{\Delta}_{e 2}^{*}$. This implies that there are disturbance vector fields for which the DDDP is solvable by applying Singh's algorithm in one way, but not solvable by applying Singh's algorithm in the other way. This contradicts Theorem 2.6. Hence $\tilde{\Delta}_{e 1}^{*}$ equals $\tilde{\Delta}_{e 2}^{*}$.

Let $\tilde{\Delta}^{*}$ be defined as the projection of the distribution $\tilde{\Delta}_{e}^{*}$ on the T $\mathscr{X}$-space. Note that, since (42) holds, $\tilde{\Delta}^{*}$ is a well-defined distribution on $\mathscr{X}$ (cf. [21]). Then $\tilde{\Delta}^{*}$ contains all possible vector fields that can be decoupled from the outputs by dynamic state feedback.

For the DDDPdm the reasoning is slightly different, although it follows the same lines. Here, we apply Algorithm 4.1 starting from the (not necessarily involutive) distribution $\Delta_{0}=\Delta_{e \mathscr{G}}^{*}:=\Delta_{e}^{*}+\mathscr{G} \times\{0\}$, resulting in the distribution $\tilde{\Delta}_{e \mathscr{G}}^{*}:=\Delta_{k^{*}}$. Analogously to Lemma 4.3, it is then possible to prove Lemma 4.4.

LEMMA 4.4. $\tilde{\Delta}_{e c g}^{*}$ obtained in the way described above is independent of the way we apply Singh's algorithm.

Now let $\tilde{\Delta}_{\mathscr{G}}^{*}$ be defined as the projection of the distribution $\tilde{\Delta}_{e^{\mathscr{G}}}^{*}$ on the $T \mathscr{X}$-space. Again, this is a well-defined distribution on $\mathscr{X}$ (cf. [21]). It is easy to see that $\tilde{\Delta}_{\mathscr{G}}^{*}$ contains all possible vector fields that can be decoupled from the outputs by dynamic state feedback with disturbance measurements. Note that in particular $\mathscr{G} \subset \tilde{\Delta}_{\mathscr{G}}^{*}$. Summarizing, we have the following theorem. 
THEOREM 4.5. Consider the system $\Sigma$ and assume that it satisfies (A1), (A2). Let $x_{0}$ be a strongly regular point for $\Sigma$. Then

1. The DDDPdm is locally solvable around $x_{0}$ if and only if

$$
\mathscr{P} \subset \tilde{\Delta}_{\mathscr{G}}^{*} .
$$

2. The DDDP is locally solvable around $x_{0}$ if and only if

$$
\mathscr{P} \subset \tilde{\Delta}^{*} \text {. }
$$

In the theorem above, we have given geometric conditions under which the DDDPdm is locally solvable. Now, of course, the question arises as to when the problem is solvable by means of a static state feedback. For this we will have to calculate $\Delta^{*}$, the maximal locally controlled invariant distribution for $\Sigma$ contained in ker $\mathrm{d} h$. Obviously, $\Delta^{*}$ is contained in $\tilde{\Delta}^{*}$, because $\Delta^{*}$ consists of the set of disturbance vector fields that can be decoupled by static state feedback. Observing that also $\tilde{\Delta}^{*}$ is contained in ker $\mathrm{d} h$, it is easily seen that $\Delta^{*}$ is the maximally locally controlled invariant distribution contained in $\tilde{\Delta}^{*}$. Hence $\Delta^{*}$ can be calculated by applying the Controlled Invariant Distribution Algorithm (cf. [12]) starting from $\tilde{\Delta}^{*}$.

Remark 4.6. Note that if the dimension of $\Delta^{*}$ equals the dimension of the zero dynamics manifold, then the DDDP is solvable if and only if the DDP is solvable.

Theorem 4.5 applied to the example of $\S 2$ yields the following result.

Example 4.7. Consider again the system of Example 2.2. Choose $\tilde{y}_{1}=y_{1}$. Then

$$
\Delta_{e}^{*}=\operatorname{span}\left\{\frac{\partial}{\partial x_{5}}, x_{2}\left(x_{2}+z\right) \frac{\partial}{\partial x_{2}}-\left(x_{2}^{2}-x_{4} z\right) \frac{\partial}{\partial x_{4}}\right\}
$$

and

$$
\tilde{\Delta}^{*}=\operatorname{span}\left\{\frac{\partial}{\partial x_{5}}\right\} .
$$

5. Example. Consider the voltage frequency controlled induction motor that was described in [3]. As state variables we take the projections of the stator current and flux vectors on a reference frame $(\alpha, \beta)$, which is fixed to the stator windings, and the angular position of the voltage input vector. As inputs we take the amplitude of the voltage input vector and the voltage supply frequency. The parameters $R_{s}$ and $R_{r}$ are the stator and rotor resistances, $L_{s}$ and $L_{r}$ are the stator and rotor self-inductances, and $M$ is the mutual inductance. The speed $\omega$ can be considered as a slowly varying parameter, due to the large separation of timescales between the mechanical and the electromagnetic dynamics. In the following, we will assume it to be constant.

We define $\bar{x}=\left(x_{1}, \cdots, x_{4}\right)$ and $x=\left(\bar{x}, x_{5}\right)$, and we assume that a one-dimensional disturbance $q$ influences the dynamics through the disturbance vector field $p(x)=$ $\left(\begin{array}{lllll}x_{3} & x_{4} & 0 & 0 & 0\end{array}\right)^{T}$. Then the state equations are written as

$$
\dot{x}=\left(\begin{array}{c}
A \bar{x} \\
0
\end{array}\right)+\left(\begin{array}{cc}
g_{1}\left(x_{5}\right) & 0 \\
0 & 1
\end{array}\right)\left(\begin{array}{l}
u_{1} \\
u_{2}
\end{array}\right)+p(x) q
$$

where

$$
A=\left(\begin{array}{cccc}
-(\alpha+\beta) & -\omega & \beta / L_{s} & \omega / \sigma L_{s} \\
\omega & -(\alpha+\beta) & -\omega / \sigma L_{s} & \beta / L_{s} \\
-\alpha \sigma L_{s} & 0 & 0 & 0 \\
0 & -\alpha \sigma L_{s} & 0 & 0
\end{array}\right), \quad g_{1}\left(x_{5}\right)=\left(\begin{array}{c}
\cos x_{5} / \sigma L_{s} \\
\sin x_{5} / \sigma L_{s} \\
\cos x_{5} \\
\sin x_{5}
\end{array}\right)
$$

and $\alpha=R_{s} / \sigma L_{s}, \beta=R_{r} / \sigma L_{r}, \sigma=1-\left(M^{2} / L_{s} L_{r}\right)$. 
Suitable outputs for the system are defined in terms of the stator flux and the torque. Hence, the following nonlinear output functions will be used:

$$
\begin{aligned}
& h_{1}(x)=\Phi_{s}^{2}=x_{3}^{2}+x_{4}^{2}, \\
& h_{2}(x)=T_{m}=x_{2} x_{3}-x_{1} x_{4} .
\end{aligned}
$$

Applying the Controlled Invariant Distribution Algorithm (cf. [12]), using REDUCE, we find that $\Delta^{*}=\{0\}$. Hence neither DDPdm nor DDP is solvable for (47), (49). However, by applying Singh's algorithm to (47), (49), we find that we can solve the DDDPdm by applying the following Singh compensator with disturbance feedthrough:

$$
\begin{aligned}
\dot{z} & =v_{1}, \\
u_{1} & =\phi_{1}(x, z), \\
u_{2} & =\phi_{2}\left(x, z, q, v_{1}, v_{2}\right),
\end{aligned}
$$

where

$$
\phi_{1}(x, z)=\frac{2 \alpha \sigma L_{s}\left(x_{1} x_{3}+x_{2} x_{4}\right)+z}{2\left(x_{3} \cos x_{5}+x_{4} \sin x_{5}\right)}
$$

and where $\phi_{2}\left(x, z, q, v_{1}, v_{2}\right)$ can be calculated from

$$
\begin{aligned}
\phi_{2}\left(x, z, q, v_{1}, v_{2}\right)= & \frac{1}{\mathscr{L}_{g_{2}} \mathscr{L}_{f} y_{2}+\phi_{1} \mathscr{L}_{g_{2}} \mathscr{L}_{g_{1}} y_{2}}\left[v_{2}-\frac{\partial \phi_{1}}{\partial z} \mathscr{L}_{g_{1}} y_{2} \cdot v_{1}\right. \\
& -\left(\mathscr{L}_{f+\phi_{1} g_{1}} \mathscr{L}_{f} y_{2}+\mathscr{L}_{g_{1}} y_{2} \mathscr{L}_{f+\phi_{1} g_{1}} \phi_{1}+\phi_{1} \mathscr{L}_{f+\phi_{1} g_{1}} \mathscr{L}_{g_{1}} y_{2}\right) \\
& \left.-q\left(\mathscr{L}_{p} \mathscr{L}_{f} y_{2}+\mathscr{L}_{g_{1}} y_{2} \mathscr{L}_{p} \phi_{1}+\phi_{1} \mathscr{L}_{p} \mathscr{L}_{g_{1}} y_{2}\right)\right] .
\end{aligned}
$$

6. Conclusions. In this paper we have introduced the dynamic disturbance decoupling problem (DDDP) for nonlinear systems, and a local solution is obtained in the case where the system under consideration is invertible. The solution is given in both an algebraic and a (differential) geometric way. This clearly exhibits that the DDDP forms a proper extension of the standard (static) DDP (cf. [13]). As stated our solution is obtained for invertible (square) nonlinear systems. An extension of the results in this paper to nonsquare, noninvertible systems can be found in [10].

Acknowledgments. The authors would like to thank A. J. van der Schaft for some very useful discussions and suggestions. The second author gratefully acknowledges some very useful preliminary discussions with Prof. U. Kotta on the topic of this paper. Finally, we have learned that very recently W. Respondek (Warsaw) has obtained similar results on the DDDP using a different type of dynamic compensator than ours.

\section{REFERENCES}

[1] G. BASILE AND G. MARRO, Controlled and conditioned invariant subspaces in linear system theory, J. Optim. Theory Appl., 3 (1969), pp. 306-315.

[2] S. P. Bhattacharyya, Disturbance rejection in linear systems, Internat. J. Control, 5 (1974), pp. 633637.

[3] A. De LucA AND G. Ulivi, Dynamic decoupling of voltage frequency controlled induction motors, in Analysis and Optimization of Systems, A. Bensoussan and J. L. Lions, eds., Lecture Notes in Control and Inform. Sci., 111, Springer, Berlin, 1988, pp. 127-137. 
[4] M. D. DI BENEDetTo AND J. W. GRIzZle, Intrinsic notions of regularity for local inversion, output nulling, and dynamic extension of nonsquare systems, Control Theory Adv. Tech., 6 (1990), pp. 357-381.

[5] M. D. Di Benedetto, J. W. Grizzle, ANd C. H. Moog, Rank invariants of nonlinear systems, SIAM J. Control Optim., 27 (1989), pp. 658-672.

[6] M. D. DI BENEDETTO AND A. ISIDORI, The matching of nonlinear models via dynamic state feedback, SIAM J. Control Optim., 24 (1986), pp. 1063-1075.

[7] A. GlumineAu AND C. H. Moog, The essential orders and the nonlinear decoupling problem, Internat. J. Control, 50 (1989), pp. 1825-1834.

[8] R. M. HIRSCHORN, Invertibility of multivariable nonlinear control systems, IEEE Trans. Automat. Control, AC-24 (1979), pp. 855-865.

[9] - $(A, B)$-invariant distributions and disturbance decoupling of nonlinear systems, SIAM J. Control Optim., 19 (1981), pp. 1-19.

[10] H. J. C. Huijberts, H. NiJmeijer, AND L. L. M. VAN Der Wegen, Dynamic disturbance decoupling for nonlinear systems: The nonsquare and noninvertible case, in Controlled Dynamical Systems, B. Bonnard, B. Bride, J. P. Gauthier, and I. Kupka, eds., Birkhäuser, Boston, 1990, to appear.

[11] — Minimality of dynamic input-output decoupling for nonlinear systems, preprint, submitted for publication.

[12] A. IsIdORI, Nonlinear Control Systems, 2nd ed., Springer, Berlin, 1989.

[13] A. Isidori, A. J. Krener, C. Gori-Giorgi, And S. Monaco, Nonlinear decoupling via feedback: A differential geometric approach, IEEE Trans. Automat. Control, AC-26 (1981), pp. 331-345.

[14] C. H. Moog, Nonlinear decoupling and structure at infinity, Math. Control Signals Systems, 1 (1988), pp. 257-268.

[15] - Note on the left-invertibility of nonlinear systems, in Analysis and Control of Nonlinear Systems, C. I. Byrnes, C. F. Martin, and R. E. Saeks, eds., Elsevier, Amsterdam, 1988, pp. 469-474.

[16] C. H. Moog, A. M. Perdon, AND G. CONTE, Model matching and factorization for nonlinear systems: A structural approach, SIAM J. Control Optim., 29 (1990), pp. 769-785,

[17] H. NiJMeIJer AND J. M. SCHUMACHER, Zeros at infinity for affine nonlinear control systems, IEEE Trans. Automat. Control, AC-30 (1985), pp. 566-573.

[18] H. NiJmeijer ANd A. J. VAN Der Schaft, Nonlinear Dynamical Control Systems, Springer, New York, 1990.

[19] S. N. SINGH, A modified algorithm for invertibility in nonlinear systems, IEEE Trans. Automat. Control, AC-26 (1981), pp. 595-598.

[20] - Generalised decoupled-control synthesis for invertible nonlinear systems, IEE Proceedings, Vol. 128, Pt.D., 1981, pp. 157-161.

[21] A. J. VAN DER SCHAFT, Observability and controllability for smooth nonlinear systems, SIAM J. Control Optim., 20 (1982), pp. 338-354.

[22] J. W. VAN DER WOUDE, On the structure at infinity of a structured system, Report BS-R89, Centre for Mathematics and Computer Science, Amsterdam, the Netherlands, 1989.

[23] W. M. Wonham, Linear Multivariable Control: A Geometric Approach, 3rd ed., Springer, New York, 1985. 